\title{
Selective retina therapy: toward an optically controlled automatic dosing
}

\author{
Eric Seifert \\ Jan Tode \\ Amelie Pielen \\ Dirk Theisen-Kunde \\ Carsten Framme \\ Johann Roider \\ Yoko Miura \\ Reginald Birngruber \\ Ralf Brinkmann
}




\title{
Selective retina therapy: toward an optically controlled automatic dosing
}

\author{
Eric Seifert, ${ }^{a, \star}$ Jan Tode, ${ }^{b}$ Amelie Pielen, ${ }^{c}$ Dirk Theisen-Kunde, ${ }^{a}$ Carsten Framme,,${ }^{c}$ Johann Roider, ${ }^{b}$ \\ Yoko Miura, ${ }^{a, d}$ Reginald Birngruber, ${ }^{d}$ and Ralf Brinkmann ${ }^{a, d}$ \\ ${ }^{a}$ Medizinisches Laserzentrum Lübeck $\mathrm{GmbH}$, Lübeck, Germany \\ bUniversitätsklinikum Schleswig-Holstein, Klinik für Ophthalmologie, Arnold-Heller-Strasse, Kiel, Germany \\ 'Universitätsklinik für Augenheilkunde, Medizinische Hochschule Hannover, Hannover, Germany \\ dUniversität zu Lübeck, Institut für Biomedizinische Optik, Lübeck, Germany
}

\begin{abstract}
Selective retina therapy (SRT) targets the retinal pigment epithelium (RPE) with pulsed laser irradiation by inducing microbubble formation (MBF) at the intracellular melanin granula, which leads to selective cell disruption. The following wound healing process rejuvenates the chorio-retinal junction. Pulse energy thresholds for selective RPE effects vary intra- and interindividually. We present the evaluation of an algorithm that processes backscattered treatment light to detect MBF as an indicator of RPE cell damage since these RPE lesions are invisible during treatment. Eleven patients with central serous chorioretinopathy and four with diabetic macula edema were treated with a SRT system, which uses a wavelength of $527 \mathrm{~nm}$, a repetition rate of $100 \mathrm{~Hz}$, and a pulse duration of $1.7 \mu \mathrm{s}$. Fifteen laser pulses with stepwise increasing pulse energy were applied per treatment spot. Overall, 4626 pulses were used for algorithm parameter optimization and testing. Sensitivity and specificity were the metrics maximized through an automatic optimization process. Data were verified by fluorescein angiography. A sensitivity of 1 and a specificity of 0.93 were achieved. The method introduced in this paper can be used for guidance or automatization of microbubble-related treatments like SRT or selective laser trabeculoplasty. () 2018 Society of Photo-Optical Instrumentation Engineers (SPIE) [DOI: 10.1117/1.JBO.23.11.115002]
\end{abstract}

Keywords: selective retina therapy; lasers in medicine; ophthalmology; retinal pigment epithelium; selectivity; algorithm.

Paper 180263RR received May 7, 2018; accepted for publication Oct. 4, 2018; published online Nov. 3, 2018.

\section{Introduction}

\subsection{Laser Treatment of the Retina}

Retinal laser treatments have been a standard form of care for a variety of posterior segment ocular diseases for decades. ${ }^{1-3}$ Irradiation times in the lower millisecond regime are preferred. ${ }^{4,5}$ Apart from the therapeutic benefit the thermal damage induced by the appropriate laser power leads to permanent damage if the neural retina. However, many therapeutically desired processes are considered to be activated through retinal pigment epithelium (RPE) regeneration, ${ }^{6,7}$ rather than the destruction of the neural retina. Hence, a treatment designed to selectively target the RPE while sparing the neural retina from thermal damage is desired. This can be achieved through an application of laser pulses that are short enough to confine the deposited heat in the RPE. ${ }^{8,9}$ Laser pulse duration in the lower microsecond or nanosecond regime and low repetition rates are most suitable for these demands.

\subsection{Selective Retina Therapy}

Selective retina therapy (SRT) devices usually make use of a series of 15 to 30 pulses with a duration of $1.7 \mu \mathrm{s} /$ pulse applied with a repetition rate of $100 \mathrm{~Hz}{ }^{10-13}$ Under these conditions, peak temperatures are localized at and around the intracellular absorbers (melanosomes). At these hotspots, vaporization leads to microbubble formation (MBF) if the radiant exposure is high enough. This MBF occurs at a lower pulse energy than protein denaturation. ${ }^{14}$ The mechanical cell damage induced by microbubbles affects the RPE cell selectively, if the pulse energy is kept close above the nucleation threshold. ${ }^{15}$

\subsection{Consequences of Microbubble Based Cell Damage}

Since cell damage takes place just inside the RPE, no ophthalmoscopically visible changes arise in the case of selective RPE damage. ${ }^{16}$ Hence, it is impossible for the treating clinician to recognize selective RPE damage ophthalmoscopically. The only means of gaining information about sufficient dosing is through invasive fluorescein angiography (FLA), which is done post-treatment. This is a common method used to gain information about the state of the $\mathrm{RPE}^{8,17,18}$ by testing its bloodretina-barrier properties. A fluorescent dye can only leak from a choroid to the retina at defective barriers. This method is reasonable for application in clinical studies since it provides information about intended RPE lesions, but it is unreasonable for clinical daily use because it is timely and costly, and requires retreatment in the case that the dosing was not sufficient. Moreover, fluorescein injection may induce allergic reactions that are occasionally severe. ${ }^{19,20}$ A microbubble detection mechanism can serve to account for intra- and inter-individual variations of the required pulse energy for MBF and make FLA abdicable. 


\subsection{Microbubble Detection Techniques}

There are different optical ${ }^{21-24}$ and acoustical ${ }^{25}$ techniques to detect microbubbles as indicators of RPE cell damage. One approach is based on the evaluation of pressure waves emitted during the formation and dynamics of microbubbles. Tissue properties such as local melanin particle distribution (among others) within the irradiated area are altered every time microbubbles are induced. This leads to slight changes in the optoacoustic response. These changes are evaluated through the optoacoustic feedback approach. This technique, introduced by Schuele et al., ${ }^{25}$ is already being applied clinically. ${ }^{11,12}$ For a reliable detection of microbubbles with the current optoacoustic technique, 30 pulses of the same pulse energy are required.

Another experimental approach is based on an evaluation of bubble-related tissue displacement through optical coherence tomography. ${ }^{23,24} \mathrm{~A}$ further experimental optical approach makes use of the evaluation of the backscattered light of a scanned laser beam. In this case, an increase in backscattered treatment light, induced by additional Mie-scatterers (microbubbles), is detected. ${ }^{22}$

\subsection{Pulsed Optical Microbubble Detection Technique}

We were motivated to develop a reliable microbubble detection system, which can be used as a real-time feedback system to cease laser radiation upon bubble detection. This allows irradiation-ceasing procedures to take place. Such a system can be operated with a train of pulses of increasing pulse energy. ${ }^{21}$ The technique applied here makes use of a coherent imaging system to observe the treated area. The backscattered treatment light is used for this imaging process. In the case of no MBF, the scattering properties of the tissue do not change during the irradiation time [Fig. 1(a)]. The temporal shape of the backscattered light is equal to those of the incoming light. Figure 1(b) shows a typical example of backscattered light measured by a photodiode in the case of no MBF. In the case of MBF, the scattering properties are altered. This can be caused by the microbubble itself and by random displacements of other scatterers [Fig. 1(c)]. Microbubble-characteristic "modulations" can be found in the sensor data in those cases [Fig. 1(d)]. The optical microbubble detection technique presented in this paper is designed to identify those "fingerprints" of microbubbles to gain information about their existence.

In stable in vivo conditions (anesthetized animal, healthy fundus, same fundus regions, same breed, etc.), this technique has been successfully applied to cease laser irradiation as soon as microbubbles are detected. ${ }^{21}$ Studies on patients with central serous chorioretinopathy (CSCR) and diabetic macula edema (DME) suggest that this approach can work in clinical treatments as well. ${ }^{26,27}$

\subsection{Purpose of this Work}

The purpose of this work is to develop an algorithm that is able to discriminate sensor data, originating from laser irradiations with and without MBF, in order to gain information about RPE damage. The algorithm is expected to provide reliable information about the existence of bubble formation for each pulse. Dependent statistical probabilities that correctly discriminate sensor data were used to quantify the performance of the algorithm. The values of the performance metrics vary with the algorithm settings. Adequate algorithm settings to detect microbubble-induced modulations in sensor data were evaluated through an automated optimization procedure. The adequacy of these settings is given when the algorithm can be applied to other datasets it is not optimized with. In an ideal case, the performance metrics are identical.

The algorithm presented in this work does not represent the algorithm implemented in the R:GEN device.

\section{Material and Methods}

\subsection{Laser and Optical Detection}

In this study, the R:GEN SRT-System (Lutronic Corp., Republic of Korea) was used. This system is based on a frequency (a)

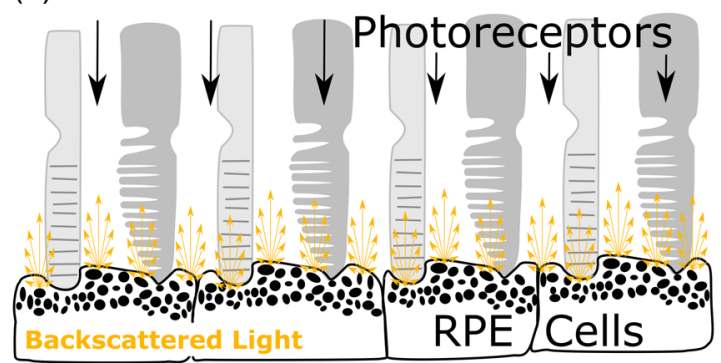

(b)

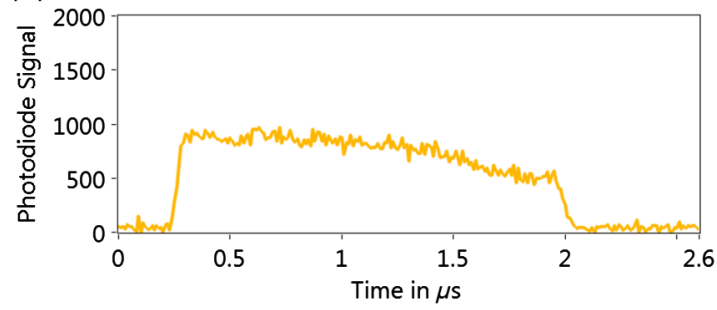

(c)

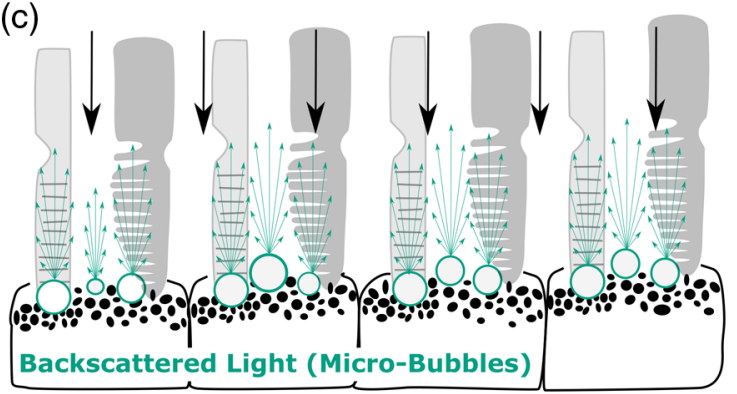

(d)

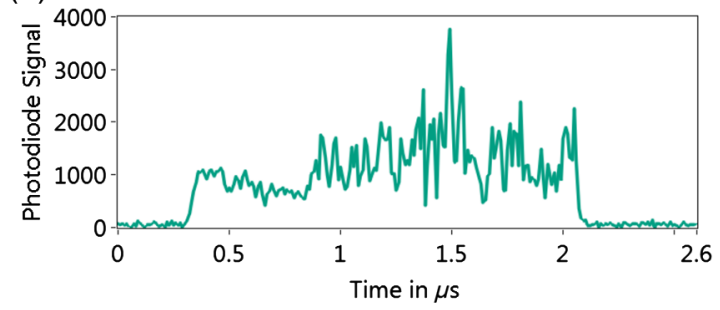

Fig. 1 (a) and (c) A schematic of irradiated RPE below and above MBF threshold. (b) and (d) An example of sensor data without and with microbubble-induced modulations, respectively. 


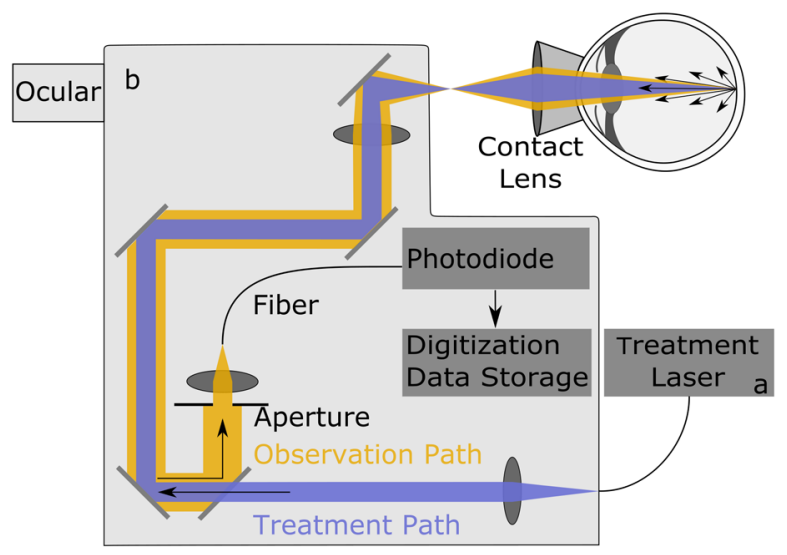

Fig. 2 Schematic of the clinical setup, highlighting the treatment path (blue) and the observation path (yellow).

doubled Q-switched Nd:YLF laser $(527 \mathrm{~nm})$ for treatment [Fig. 2(a)]. The pulse duration was set to $1.7 \mu \mathrm{s}$ and 15 pulses were applied with a repetition rate of $100 \mathrm{~Hz}$. A pulsewise increase of the laser pulse energy (ramp) was implemented (see Fig. 3). The starting energy was set to $50 \%$ of the maximum energy $E_{\max }$, chosen by the clinician. Since the laser system increases pulse energy linearly, the pulse energy step size was $3.57 \%$ of the maximum energy. All 15 pulses were always applied to each spot, and $E_{\max }$ was reached at the 15 th pulse.

The treatment light was guided to the slit lamp unit [Fig. 2(b)] via an optical step index fiber with a core diameter of $50 \mu \mathrm{m}$ and a numerical aperture of 0.11 . The fiber tip was imaged to the treatment plane with a magnification factor of 4 via two lenses. The spot diameter was $200 \mu \mathrm{m}$ in the air. Spatial irradiance variations within the beam profile were measured and had a peak-to-mean ratio of 3.5. A contact lens (R: GEN contact lens with acoustic sensor, Lutronic) with a spotmagnification of one was placed on the eye.

An observation path images the treatment spot to another fiber, guiding the backscattered light to an avalanche photodiode. An 80/20 (transmission/reflection) beam splitter separates the backscattered light. In the observation path, an aperture with a diameter of $1 \mathrm{~mm}$ affects stray light, speckle properties, and the irradiance at the photodiode. The acquired pulse is digitized with a sampling frequency of $100 \mathrm{MS} / \mathrm{s}$ and an effective resolution of 13 bit. The acquired data were stored

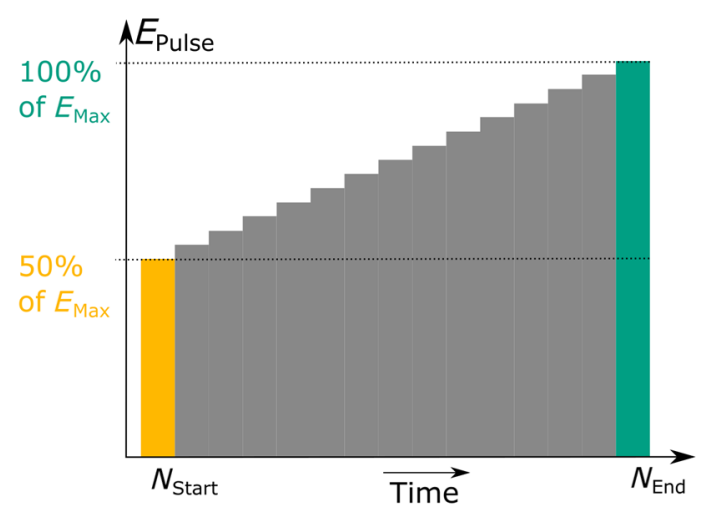

Fig. 3 Laser pulse energy ramp. The pulse energy of the first pulse (yellow) starts at $50 \%$ of the maximum energy (green), chosen by the clinician. on a hard disk. In addition to the sensor data, metainformation, such as the pulse number and the applied pulse energy, was stored. The implemented dosimetry software of the R:GEN system is very different from the software introduced here and was neither used nor analyzed in the context of this work.

\subsection{Clinical Study and Treatment}

Nineteen treatments for 15 eyes of 15 patients (gender: 10 males and 5 females; age: average 54 years old, range 35 to 76 years old) with symptomatic CSCR (11 patients) and DME (4 patients) were performed in this study. This number also includes four retreatments. The study was conducted at the eye clinics of the University Hospital Schleswig-Holstein (UKSH) Campus Kiel and Hannover Medical School (HMS). The procedures in the study adhered to the tenets of the declaration of Helsinki and its later amendments. The protocol and informed consent forms were compliant with and approved by the local institutional/ethical review boards (ethic committee vote number of HMS: 7393 and UKSH: B577/16).

At the beginning of each treatment, test irradiations were applied to spots near the arcades (test spots). The position and maximum pulse energy (or the actual number of irradiated test spot regionsl"spot number") were recorded for each spot. Figure 5(b) displays a "treatment map" documenting the test spots. The maximum laser pulse energy $\left(E_{\max }\right)$ was increased by $10 \mu \mathrm{J}$ for every second spot. The lowest $E_{\max }$ was $30 \mu \mathrm{J}$. This process was stopped as soon as ophthalmoscopically visible effects were observed. The $E_{\max }$ value used for the treatment was set to $80 \%$ of the ophthalmoscopically visible spots. To ensure selective RPE damage in the treated area and to document the state of the RPE in the test spot area, fluorescence angiography (FLA) was applied less than $1 \mathrm{~h}$ after treatment. The acquired sensor data and metadata were saved in the R-GEN system. The data were pseudonymously acquired and utilized for the analysis after this study was completed.

\subsection{Differentiation Algorithm}

A variety of different algorithms to differentiate signals with and without microbubble modulations have been developed. In this work, we present a version characterized by its low complexity. Several parameters of the algorithm are mentioned but not quantified in this section. In the development process, these parameters are evaluated through an optimization process.

Data processing is done on the digitized sensor data delivered by the analog digital converter (ADC). Initial software routines to ensure the validity of the input signals are not outlined. Figure 4(a) displays the input data for the most relevant processing steps. These processing steps are:

1. Normalization: This step serves to decouple the input data from the overall level of the backscattered light. To achieve this, the signal is normalized to the sum of its values.

2. Jitter removal: To be able to address certain regions of interest, the pulse location in the dataset needs to be constant. The routine, which ensures the fixed position of the pulse, identifies the rising flank of a pulse and extracts a subset with the pulse starting at a predefined sample. The rising flank of the pulse is found through a routine that looks for the first sample exceeding the 

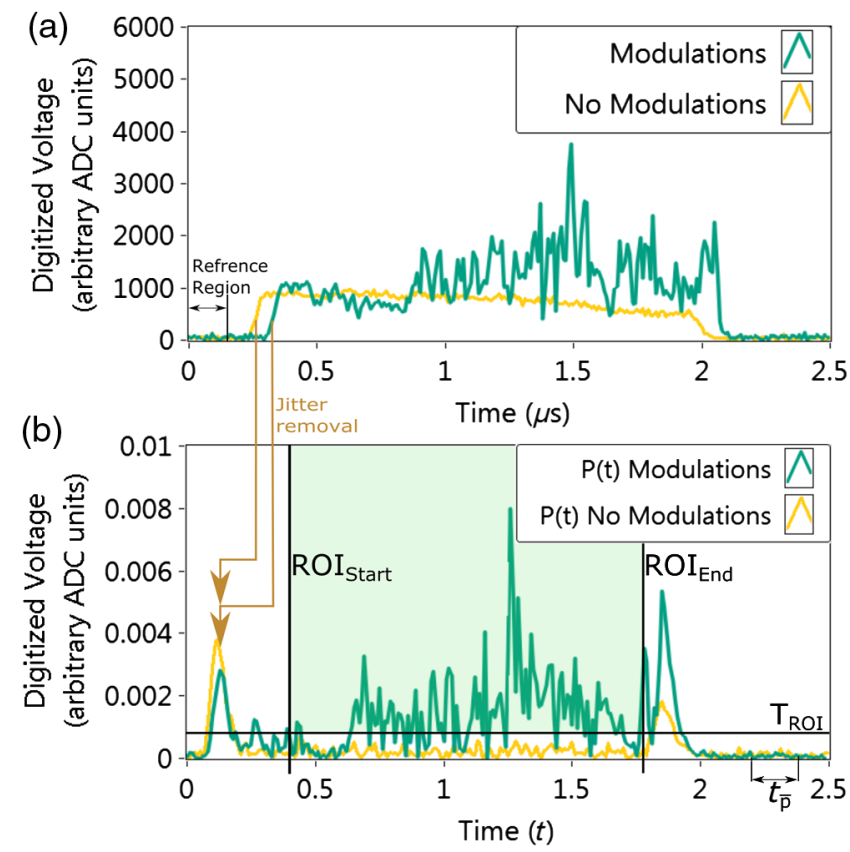

Fig. 4 (a) Signals for a scenario with and without microbubbleinduced modulation in green and yellow. (b) Jitter removed and filtered signals. The ROI to be evaluated is highlighted.

threshold $T_{\text {Jitt }}$. This threshold is a product of the mean of a reference region not belonging to the pulse [see Fig. 4(a) "reference region"] and a factor $F_{J}$, which is a variable for the optimization process of the algorithm.

3. Filtering: For the extraction of modulations, a bandpass filter (Butterworth type) is used. The values of the higher and the lower cutoff frequency $\left(f_{\mathrm{H}}\right.$ and $\left.f_{\mathrm{L}}\right)$ are evaluated through an optimization process.

4. Summation of a region of interest: Examples of the processed photodiode signal $P(t)$ are given in Fig. 4(b) for a pulse above and below the threshold in green and yellow, respectively. When a region of interest (ROI) is defined, modulations can be identified in the filtered signal. The ROI is defined by its starting point $\left(\mathrm{ROI}_{\text {Start }}\right)$, its endpoint $\left(\mathrm{ROI}_{\text {End }}\right)$, and a threshold value $T_{\mathrm{ROI}}$. The threshold value $T_{\mathrm{ROI}}$ defines a minimum value. Values above this threshold belong to the ROI.

Values within the ROI are taken into consideration by the final quantification step exclusively. Figure 4(b) illustrates these values. It can be seen that $\mathrm{ROI}_{\text {Start }}$ and $\mathrm{ROI}_{\text {End }}$ address an interval within the pulse but not the edges of the pulse. The threshold value $T_{\mathrm{ROI}}$ is defined by the mean of the filtered dataset in a region not belonging to the pulse [see Fig. 4(b), $\mathrm{t}_{\bar{p}}$ ] multiplied by the factor $F_{\mathrm{ROI}}$. The factor $F_{\mathrm{ROI}}$ is a variable for the optimization process of the algorithm. The extracted modulations can be found in the subset $P_{\mathrm{ROI}}$ :

$$
\left\{P_{\mathrm{ROI}}(t) \epsilon P(t) \mid \mathrm{ROI}_{\text {Start }} \leq t \leq \mathrm{ROI}_{\text {End }} \cap P(t)>T_{\mathrm{ROI}}\right\} .
$$

Figure 4(b) illustrates the filtered signals originating from the data displayed in Fig. 4(a). The green region highlights (a)

(b)

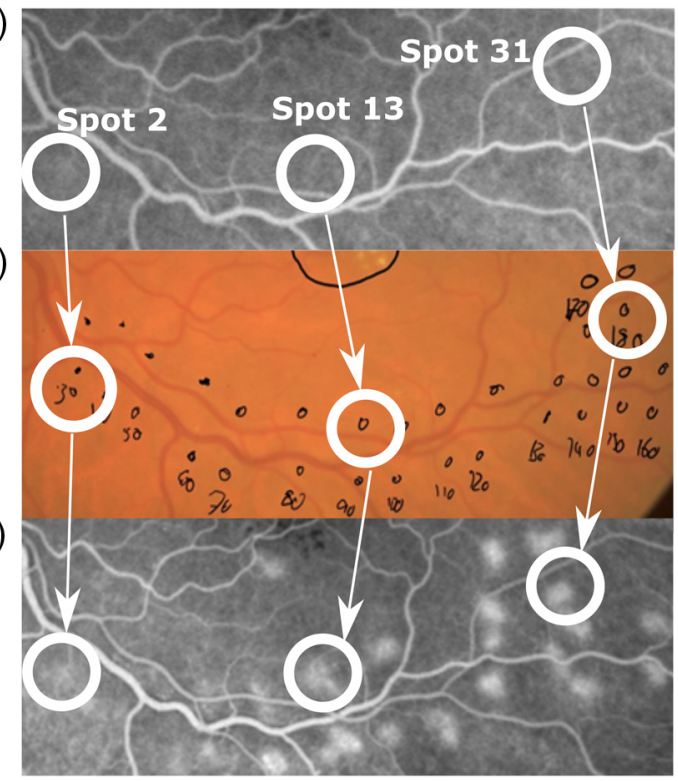

Fig. 5 Example of an FLA-nonvisible, FLA-visible, and an ophthalmoscopically visible region. (a) FLA-image taken days before treatment. (b) "Treatment map" showing the spot allocation in the irradiated test spot region. (c) FLA image taken after treatment.

the threshold ROI. The final reflectometry (RM) value is expressed by the sum of values in this ROI.

$\mathrm{RM}=\sum P_{\mathrm{ROI}}(t)$

\subsection{Classification of Data}

The acquired sensor data and metadata can be assigned to an "FLA visibility class" for each documented test spot. The spot-assigning process starts with a comparison of all identifiable spots [hyperfluorescent spots in Fig. 5(c)] to all documented spots [Fig. 5(b)]. If the locations of the visible spots match with the locations of the documented spots, the nonFLA-visible spots are assumed to be located at the documented regions as well. If there is a mismatch between documented spots and FLA-visible spots, FLA information is labeled as "unknown" for all spots of this treatment. Furthermore, it needs to be possible to distinguish a selective RPE lesion from its surroundings. If this is not possible (e.g., due to intense pathological leakage), all spots of this treatment are labeled as "unknown" with regard to their FLA information. If those impediments do not exist, the FLA information can be labeled as "FLA-visible" or "FLA-nonvisible."

In addition to the FLA classification, each test spot is assigned to an "ophthalmoscopic visibility class." Information about ophthalmoscopic visibility is provided by a note on the treatment maps.

In the case of a FLA-nonvisible spot, all 15 pulses were classified as FLA-nonvisible. In the case of FLA-visible or ophthalmoscopically visible spots, it is unknown which pulses contributed to the corresponding visibility. Due to this attribution problem, the pulse with the highest RM value (per spot) was used for FLA-classification and the pulse with the highest pulse energy was considered for ophthalmoscopic visibility classification.

In addition to the spotwise classification of FLA and ophthalmoscopic visibility, each single pulse of each spot was classified 


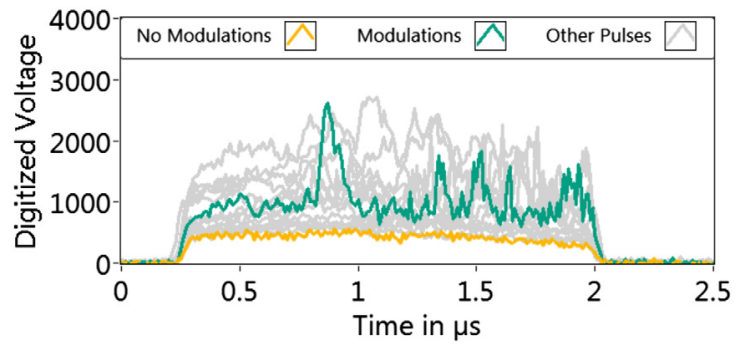

Fig. 6 Sensor data classification. The yellow pulse is classified as "no modulations" and the green pulse is classified as "modulations."

according to the (non)existence of microbubble-induced characteristics (modulations) in its sensor data. A classification according to this "modulation class" is done for both test and treatment spots. In the first step of this classification process, an observer subjectively decides whether a microbubble-induced signal can be detected or not. If this is not possible (e.g., low by signal to noise ratio), the pulse is labeled as "unknown." Otherwise, a binary classification (modulation/no modulation) is assigned. Examples of pulses with "no modulation" can be found in Fig. 6 (yellow). Examples of pulses with microbubble-induced modulation can be found in Fig. 6 (red).

\subsection{Datasets}

Information about the generalizability of the achieved performance can be gained by comparing the performance of the dataset used to evaluate the algorithm settings with other datasets. Overall, there are three datasets.

Data in the "training dataset" originate from test spots that could not be evaluated according to FLA imaging (FLAclassification "unknown") and the treatment spots. Data from treatment spots were labeled with modulation class information only. The training dataset is the only dataset used for the automatic optimization process.

In the evaluation process, the performance metrics of the training dataset were compared to the so-called cross-validation set (CV set). Data from the CV set originate from patients whose data have not been applied for training. The data of the CV set fulfill the same criteria as the training set data. There are 15 treatments for 12 patients (nine CSCR, three DME) in the training set and four treatments of three patients (two CSCR, one DME) in the CV set.

Comparisons with regard to sensitivity and specificity values between the training and $\mathrm{CV}$ sets offer information about the generalizability of the algorithm's performance to detect microbubble-induced modulations in sensor data.

Finally, the data from the so-called "test set" originate from FLA-evaluable test spots exclusively. This data was classified according to all three categories ("modulation," "FLA visibility," and "ophthalmoscopic visibility"). Comparisons between the training and test sets offer information about the applicability of algorithmically detected modulations in sensor data to detect FLA visible RPE cell damage in test spots. Data from 11 treatments on 11 patients are included in the test set (nine CSCR, two DME).

\subsection{Performance Metrics}

The algorithm's performance is expressed through dependent statistical probabilities to correctly assign a pulse to its "modulation" or "FLA visibility" class. This is done by thresholding the RM value. The estimated dependent probabilities for correctly assigning a positive (e.g., "modulation") or negative (e.g., "no modulation") pulse to its class are called sensitivity and specificity, respectively. Sensitivity and specificity can be calculated for multiple threshold values. A $X-Y$-plot of sensitivity and specificity for each threshold value is called "receiver operating characteristic" (ROC) curve. During the optimization procedure, the area under the ROC curve (AUC) ${ }^{28}$ is maximized. The AUC was chosen as an optimization criterion since it is independent from individual thresholds. The ideal threshold is determined in a separate step through an index merging sensitivity and specificity (Y-Index). ${ }^{29}$

In optimization processes, it is not uncommon that the best performing settings are influenced by dataset-individual nuances. This so-called "over-fitting" to those nuances is not intended because the seemingly good performance would not be achieved when those settings are applied to other datasets. The performance would not be generalizable. In the context of this work, the performance metric differences (PM difference) between datasets are used as a metric of the performancegeneralizability.

\subsection{Microbubble-Detection-Based Therapeutic Window}

If the pulse energy for initial MBF is exceeded, there is a range in which a treatment can still be performed without typical indications of a damaged neural retina. In previous studies, this "therapeutic window" (TW) has been defined by the range between the metric value (e.g., pulse energy) that leads to a $50 \%$ chance of inducing FLA-visible RPE damage $\left(\mathrm{ED} 50_{\mathrm{FLA}}\right)$ and the metric value that leads to a $50 \%$ chance of ophthalmoscopically visible damage $\left(\mathrm{ED} 0_{\mathrm{Oph}}\right)$. In this work a more conservative definition was applied. The lower boundary of the TW was determined by the the metric value to achieve an $86 \%$ probability of finding FLA visible lesions $\left(\right.$ ED86 $\left._{\text {FLA }}\right)$. The upper boundary was determined by the metric value to achieve a $16 \%$ probability of finding ophthalmoscopically visible lesions $\left(\mathrm{ED} 16_{\mathrm{Oph}}\right)$. This range was called "safety range" in former animal studies. ${ }^{10}$ The ratio of the lower $95 \%$ confidence interval of the upper boundary (ED16 $6_{\text {Oph }}$ ) and the upper $95 \%$ confidence interval of the lower boundary (ED86 FLA $)$ was used to define the TW. Since the algorithm is designed and optimized to make a binary decision, not to deliver a value scaling of cell damage, a TW definition based on the pulse energy/radiant exposure is reasonable. In former studies, the TW has been defined by the ratio of absolute values of the radiant exposure.

The combination of single pulse microbubble detection with a pulse energy ramp offers the possibility of identifying a spot-specific reference energy for initial MBF at each spot. Each pulse's energy $E_{p}$ can be set in relation to such a reference point for initial MBF $\left(E_{\mathrm{Ref}}\right)$. For each spot, the first pulse leading to an RM value above RM threshold defines $E_{\text {Ref }}$ :

$\delta=\frac{E_{p}}{E_{\mathrm{Ref}}}$,

where $E_{\text {Ref }}$ cannot be calculated for FLA nonvisible spots, because those spots usually do not have pulses with microbubble modulations (thus, no RM values above RM threshold). Since this calculation cannot be done for every spot, $E_{\text {Ref }}$ was evaluated eye-wise. It is defined by the mean of the eyes' local 
Table 1 Column "optimization boundaries": boundaries the optimization variables need to stay in during the optimization process. Column "optima range:" the range of values the variables of the best performing optima accumulate in. Column "optima used": the algorithm settings applied for further discussion.

\begin{tabular}{lccc}
\hline & $\begin{array}{c}\text { Optimization } \\
\text { boundaries }\end{array}$ & Optima range & $\begin{array}{c}\text { Chosen } \\
\text { optimum }\end{array}$ \\
\hline $\begin{array}{l}\text { Jitter threshold } \\
F_{\text {Jitt }} d\end{array}$ & 1 to 10 & 3 to 8 & 4.9 \\
$\begin{array}{l}\text { Higher cutoff } \\
\text { frequency } f_{H}\end{array}$ & 16 to $50 \mathrm{MHz}$ & 25 to $42 \mathrm{MHz}$ & $25.5 \mathrm{MHz}$ \\
$\begin{array}{l}\text { Lower cutoff } \\
\text { frequency } f_{L}\end{array}$ & 1 to $15 \mathrm{MHz}$ & 3 to $9 \mathrm{MHz}$ & $3.5 \mathrm{MHz}$ \\
$\begin{array}{l}\text { ROI range } \\
\text { ROI threshold }\end{array}$ & 0.15 to $1.80 \mu \mathrm{s}$ & 0.25 to $1.74 \mu \mathrm{s}$ & 0.38 to $1.74 \mu \mathrm{s}$ \\
factor $F_{\mathrm{ROI}}$ & 1 to 10 & 3 to 6 & 5.9 \\
\hline
\end{tabular}

(spot-wise) $E_{\text {Ref }}$ values. The classified data (e.g., FLA visibility) and the normalized pulse energy $\delta$ were used for the statistic calculations (probit evaluations) required for microbubbledetection-based $\mathrm{TW}_{\delta}$. This implies that the $\mathrm{TW}_{\delta}$-defining boundaries $\left(\mathrm{ED} 16_{\mathrm{Oph}}\right.$ and $\mathrm{ED} 86_{\mathrm{FLA}}$ ) become dependent on the threshold value.

\section{Results}

\subsection{Clinical Data Overview}

Overall, 4626 pulses were used for this evaluation. This data was acquired during the treatment of 15 patients in the eye clinics of the UKSH Kiel and the Hannover Medical School. The trainingset consists of 2516 pulses classified as "modulation" and 615 pulses classified as "no modulation." The CV set consists of 1121 pulses classified as "modulation" and 165 pulses classified as "no modulation." The test set consists of 106 pulses of spots classified as "FLA visible" and 103 pulses of spots classified as "FLA-nonvisible." (a)

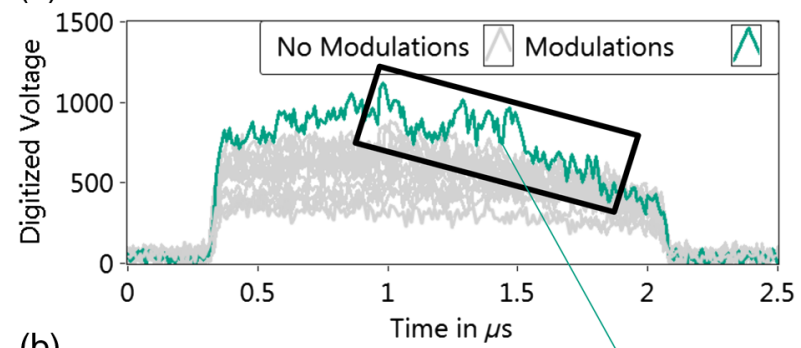

(b)

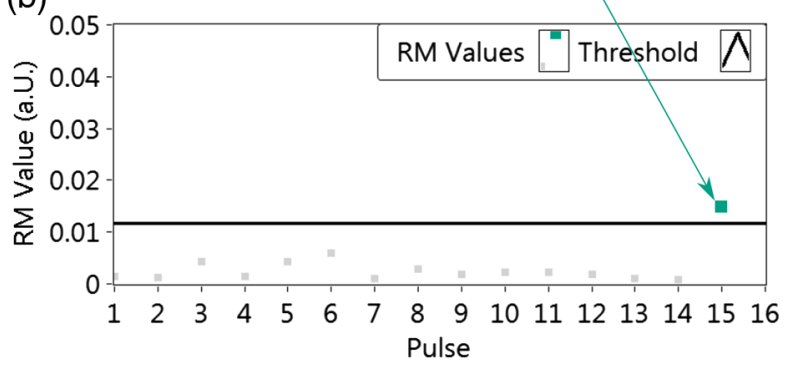

Fig. 8 (a) Pulses applied to a spot classified as FLA visible. (b) RM values of the pulses of panel $a$. The last pulse shows microbubbleinduced modulations.

\subsection{Training Set Optima}

Several optimal algorithm settings were found through the optimization procedure. The ranges of reasonable optima can be found in the third column of Table 1. For further discussion, the settings displayed in the fourth column (chosen optimum) are used.

It was found that sensor data for spots without any microbubble-induced modulations [Fig. 7(b)] led to RM value accumulation in a low-value-regime [Fig. 7(a), yellow dot]. This data can typically be found in FLA-nonvisible spots. In the case of FLA visible spots, it can often be found the first pulses do not display microbubble-induced modulations, but the latter pulses do [Fig. 7(c)]. This leads to RM values in the low-valueregion as well. However, in contrast to FLA-nonvisible spots, the RM values increase at spots with higher pulse energy (a)

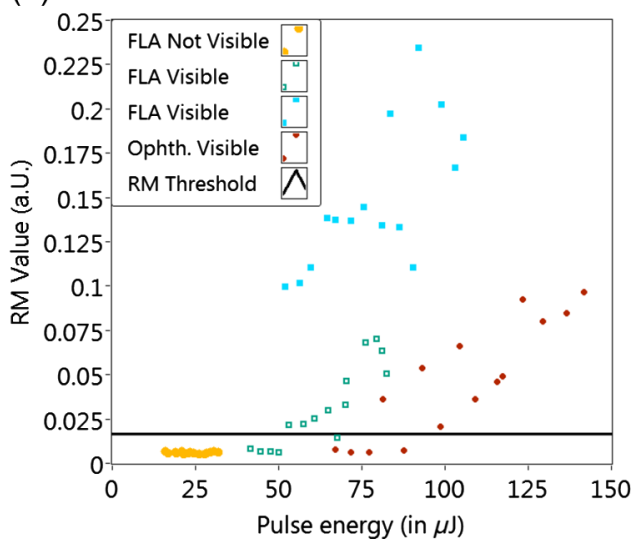

(b)

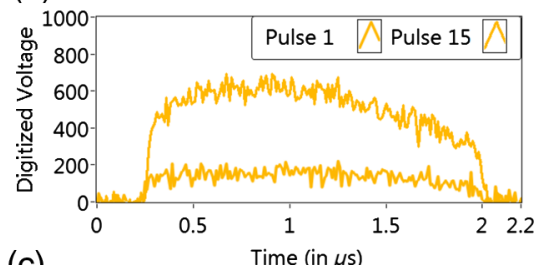

(c)

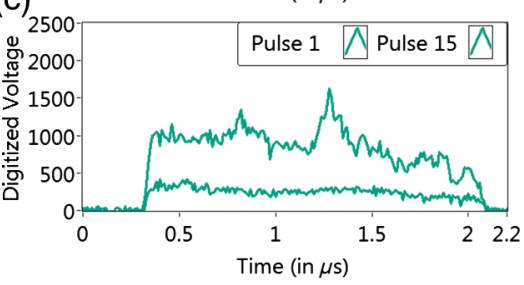

(d)

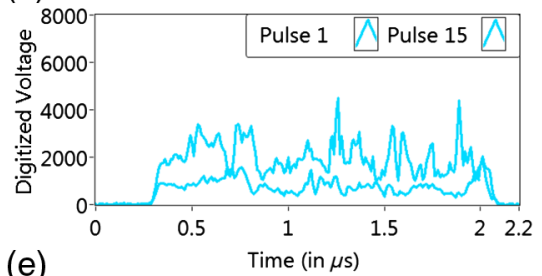

(e)

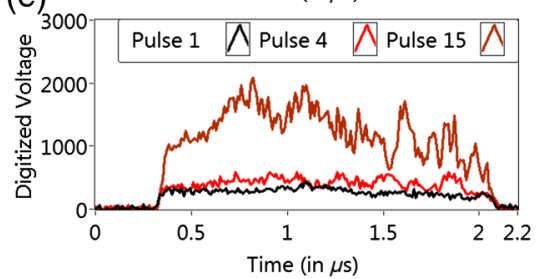

Fig. 7 (a) RM values of selected examples processed with the algorithm settings presented in Table 1 (column "Optima applied"). (b)-(e) the first and last pulses of the mentioned examples are displayed. (e) The pulse with initial microbubble modulations (pulse 4, red) is displayed as well. The examples include sensor data of FLA-nonvisible spots (b), FLA-visible spots (c and d) and ophthalmoscopically visible spots (e). 
Table 2 Performance metric value (PM) and performance metric difference (PM-difference) overview. A RM threshold of 0.012 leads to a maximum Y-index (bold number) in the CV dataset, a RM-threshold of 0.23 leads to a specificity of 1 (bold number) in the CV dataset.

\begin{tabular}{|c|c|c|c|c|}
\hline Set & $\begin{array}{l}\text { Performance metric } \\
\text { value ( } 1 \text { is optimal) }\end{array}$ & $\begin{array}{l}\text { Performance metric } \\
\text { difference ( } 0 \text { is optimal) }\end{array}$ & $\begin{array}{l}\text { CV Y-index } \\
\text { (1 is optimal) }\end{array}$ & RM threshold \\
\hline Training set sensitivity & 0.97 & 0.01 & & \\
\hline CV set sensitivity & 0.98 & & & \\
\hline Training set specificity & 0.98 & 0.005 & 0.98 & 0.012 \\
\hline CV set specificity & 0.98 & & & \\
\hline Training set sensitivity & 0.88 & 0.017 & & \\
\hline CV set sensitivity & 0.9 & & & \\
\hline Training set specificity & 1.0 & 0 & 0.9 & 0.023 \\
\hline CV set specificity & 1.0 & & & \\
\hline
\end{tabular}

[Fig. 7(a), green hollow rectangles]. In Fig. 8, an example of sensor data and the corresponding RM of initial MBF is given. Only the 15th pulse of the FLA-visible spot exceeds the threshold for MBF.

Other FLA-visible spots received maximum pulse energy far above initial MBF. In such cases, even the first pulse displays microbubble-induced modulations in the sensor data [Fig. 7(d)]. In those pulses, it cannot be predicted how far above the MBF threshold the first pulse already is. [Fig. 7(a), blue rectangles]. Since the pulse energy doubles from the first pulse to the last and since the FLA visible spots are ophthalmoscopically nonvisible, this observation implies that $\delta$ of the local pulse energy for ophthalmoscopic visibility is typically larger than two.

The same observation (first pulse above MBF threshold) can also be made in the case of ophthalmoscopically visible spots. There are only two examples of ophthalmoscopically visible spots with a first pulse without microbubble-induced modulations. One example is displayed in Fig. 7(e). At this irradiation, initial MBF occurs at $(81.4 \mu \mathrm{J})$. The corresponding pulse is plotted in red. A $E_{\max }$ of $141.7 \mu \mathrm{J}$ was measured. Thus, there is a $\delta$ of 1.74 from initial MBF to ophthalmoscopic visibility at this pulse. These spots' RM values were plotted in Fig. 7(a) (red dots). In the second evaluable ophthalmoscopically visible case $\delta$ is 1.84 .

\subsection{General Algorithmic Performance}

The performance measures (PM) as sensitivity and specificity (see Sec. 2.6) of the chosen algorithm settings (Table 1, column 4) are presented for two threshold values. The threshold value of 0.012 leads to a maximum Y-index of 0.98 in the cross-validation dataset (see upper part of Table 2 column "CV Y-index"). Sensitivity and specificity are equally close to the optimum value of one. The highest performance measure difference (PM-Difference) is 0.017 .

In SRT, it is desirable to lower the number of false positives in order to maximize a therapeutic effect. One way to achieve this is to increase the RM threshold. At a threshold of 0.023 , the CV set does not have any false positives (see lower part of Table 2 bold printed number). Increasing the specificity by
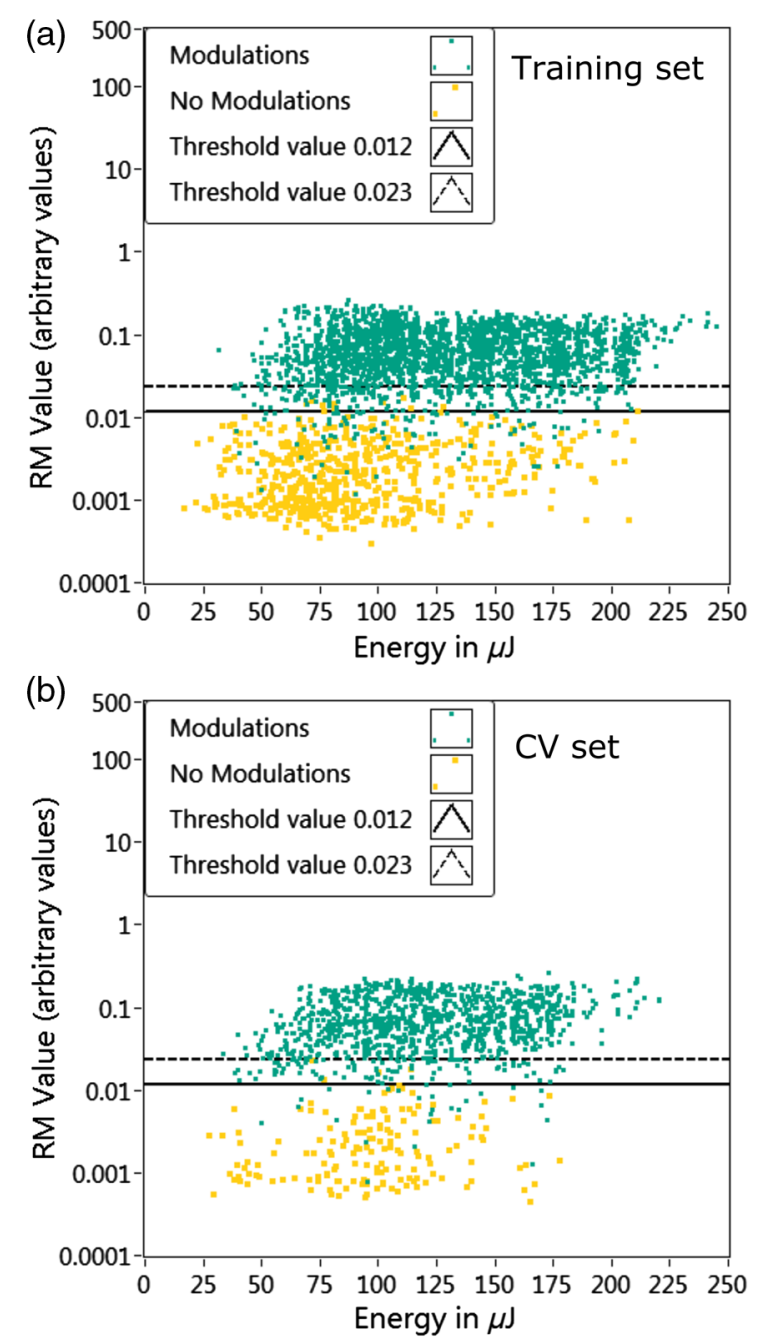

Fig. 9 Scatterplots (a) and (b) display pulsewise data of the training and CV sets, respectively. The existence of microbubble-induced modulations is color coded. 
Table 3 Performance metric value (PM) and performance metric difference (PM-difference) overview. A RM threshold of 0.012 also leads to a high $\mathrm{TW}_{\delta}$. A RM-threshold of 0.23 leads to a specificity of 0.98 in the test dataset, but a decreased $\mathrm{TW}_{\delta}$.

\begin{tabular}{|c|c|c|c|c|}
\hline Set & $\begin{array}{l}\text { Performance metric } \\
\text { value ( } 1 \text { is optimal) }\end{array}$ & $\begin{array}{l}\text { Performance metric } \\
\text { difference ( } 0 \text { is optimal) }\end{array}$ & $\begin{array}{l}\mathrm{TW}_{\delta} \text { (larger } \\
\text { is better) }\end{array}$ & $\begin{array}{l}\text { Applied } \\
\text { threshold }\end{array}$ \\
\hline Training set sensitivity & 0.97 & 0.03 & \multirow{4}{*}{1.97} & \multirow{5}{*}{0.012} \\
\hline Test set sensitivity & 1 & & & \\
\hline Training set specificity & 0.98 & 0.05 & & \\
\hline Test set specificity & 0.93 & & & \\
\hline Training set sensitivity & 0.88 & 0.10 & \multirow{4}{*}{1.85} & \\
\hline Test set sensitivity & 0.98 & & & \multirow{3}{*}{0.023} \\
\hline Training set specificity & 1 & 0.02 & & \\
\hline Test set specificity & 0.98 & & & \\
\hline
\end{tabular}

increasing the RM threshold results in a decrease in sensitivity. Overall, the Y-index is reduced by 0.08 . The sensitivity and specificity values achieved by the application of this threshold are displayed in the lower part of Table 1.

The PM differences do not exceed values of 0.01 and 0.017 for thresholds of 0.012 and 0.023 , respectively. In Figs. 9(a) and 9(b), scatterplots of calculated RM values (pulsewise) against the pulse energy are displayed for the training and CV datasets, respectively. The modulation class (classified sensor data) is color coded. The horizontal lines indicate the threshold values. The solid line represents a threshold of 0.012 (maximum Y-Index in CV set), and the dashed line represents a threshold of 0.023 (no false positives in CV set).

\subsection{Applicability to FLA Visibility}

The PM differences were larger than those between training and $\mathrm{CV}$ sets, but they do not exceed values of 0.05 and 0.1 for the respective thresholds of 0.012 and 0.023 . An increase of the threshold value leads to a decrease of $\mathrm{TW}_{\delta}$. For a threshold

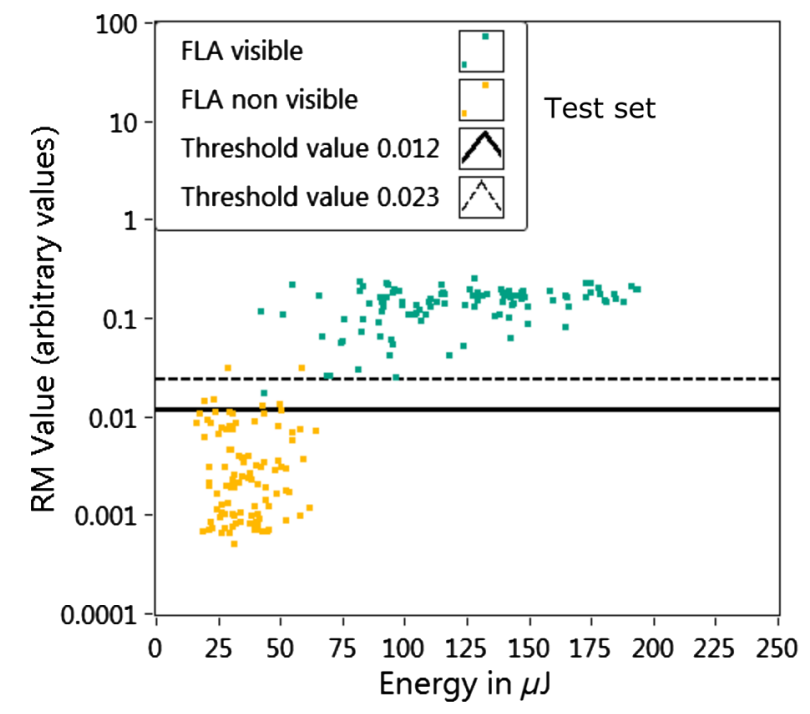

Fig. 10 The scatterplot shows test set data. The spots with highest RM values are considered. FLA visibility is color coded. of $0.012, \mathrm{TW}_{\delta}$ is 1.97 and at a threshold of $0.023 \mathrm{TW}_{\delta}$ is 1.85 . Table 3 summarizes the performance metrics, and Fig. 10 displays the scatterplots of the test set.

\subsection{Microbubble-Detection-Based Therapeutic Window and MBF Threshold Variation}

For an RM threshold value of 0.012, the ED86 energy for FLA visibility is located at factor 1.14 , above $E_{\mathrm{Ref}}$, and the ED16 for ophthalmoscopical visibility is located at factor 2.24 above $E_{\text {Ref }}$. This leads to a microbubble-detection-based $\mathrm{TW}_{\delta}=1.97$. The $\mathrm{TW}_{\delta}$ decreases to a value of 1.85 if the threshold of 0.023 is applied.

High intraindividual variations of the local energy for MBF $\left(E_{\mathrm{MBF}}\right)$ were found. Table 4 summarizes the max-min deviations of $E_{\mathrm{MBF}}$. The differences are most pronounced in patients DME2, DME3, and CSCR4. It is important to note that this evaluation is limited to nine patients only. This is due to the

Table 4 Initial MBF energy. This evaluation is limited to only nine patients due to the necessity to have at least one spot with the first pulse below the threshold for MBF to find the MBF-threshold. The RM threshold of 0.023 was applied for MBF identification. Cases with the highest Min-Max Differences are highlighted with bold numbers.

\begin{tabular}{lccc} 
Pseudonym & Mean MBF energy $(\mu \mathrm{J})$ & Min $(\mu \mathrm{J})$ & Max $(\mu \mathrm{J})$ \\
\hline CSCR 1 & 62 & 55 & 68 \\
CSCR 2 & 64 & 47 & 87 \\
CSCR 4 & 90 & 58 & 141 \\
CSCR 6 & 61 & 55 & 70 \\
CSCR 8 & 60 & 48 & 81 \\
CSCR 9 & 40 & 30 & 48 \\
CSCR 10 & 49 & 41 & 61 \\
DME 2 & $\mathbf{7 6}$ & $\mathbf{2 8}$ & $\mathbf{1 2 3}$ \\
DME 3 & 63 & 39 & $\mathbf{1 0 9}$ \\
\hline
\end{tabular}


additional constraint of having at least one spot with the first pulse below the threshold for MBF.

\section{Discussion}

Up to now, probing procedures to prove successful RPE damage have been used in SRT studies. These procedures rely on the assumption that pulse energy thresholds found in the symptomatic-free test spot region are at least similar to those in the symptomatic treatment region. Time-consuming FLA images are required to be taken after treatment since this assumption is not always met.

Microbubbles are assumed to be the origin of the modulations observed in the present sensor data and the origin of RPE cell damage. ${ }^{9,21}$ It was the goal of this study to develop and test an algorithm to discriminate data originating from spots, where microbubbles have been induced by the laser treatment from those without microbubble occurrence.

\subsection{Correlation RM Signal to FLA}

The results chapter shows that microbubble-induced-modulations in sensor data and FLA visibility are strongly related to each other. Measures for the generalizability of algorithmic performance (PM-Difference) were presented for two threshold values. The PM differences between training and test set are larger than those between training and CV set. This raises the question of the origin of this increase. Differences in the performance of microbubble detection between the healthy test region and the diseased treatment region need to be considered as well as differences in the RM value distribution. In order to test the ability to detect microbubbles in test spot regions, all FLA evaluable test spots were evaluated according to their modulation class, too. It has been found that sensitivity and specificity are comparable between training, CV, and test set. The PM difference between training and CV set is the same as the PM difference for training and test set. For both, the difference is 0.1 , assuming an RM threshold of 0.012. In Fig. 11(a), the sensitivity/specificity pairs at the RM threshold of 0.012 are highlighted for each dataset.

It can be assumed that differences in the distribution of RM values as well as difficulties associated with the classification process of FLA images may be the source of those differences. A different distribution of RM values in the test set most likely originates from the systematic application of test spots (increase of $E_{\max }$ with every second spot until ophthalmoscopically visible lesions appear). Together with the necessity to choose the highest RM value for FLA classification (FLA classification only be done spot-wise; not pulse-wise), this leads to an increased probability of high RM values. In future studies, this mismatch can be reduced by applying automatic irradiation ceasing based on the decision of the presented algorithm and threshold in the test spot area. This would result in an increased amount of RM values closer to the RM threshold for FLA evaluable spots.

\subsection{Causes of False Positives and False Negatives (Test Set)}

For further discussion, false positives and false negatives are of concern. False negatives are those datasets with signs of bubble formation (FLA-visible) but an incorrect algorithmic assignment to the opposite class (FLA-nonvisible). These cases can lead to unwanted and potentially dangerous overtreatments. There are no false negatives in the test dataset if the threshold
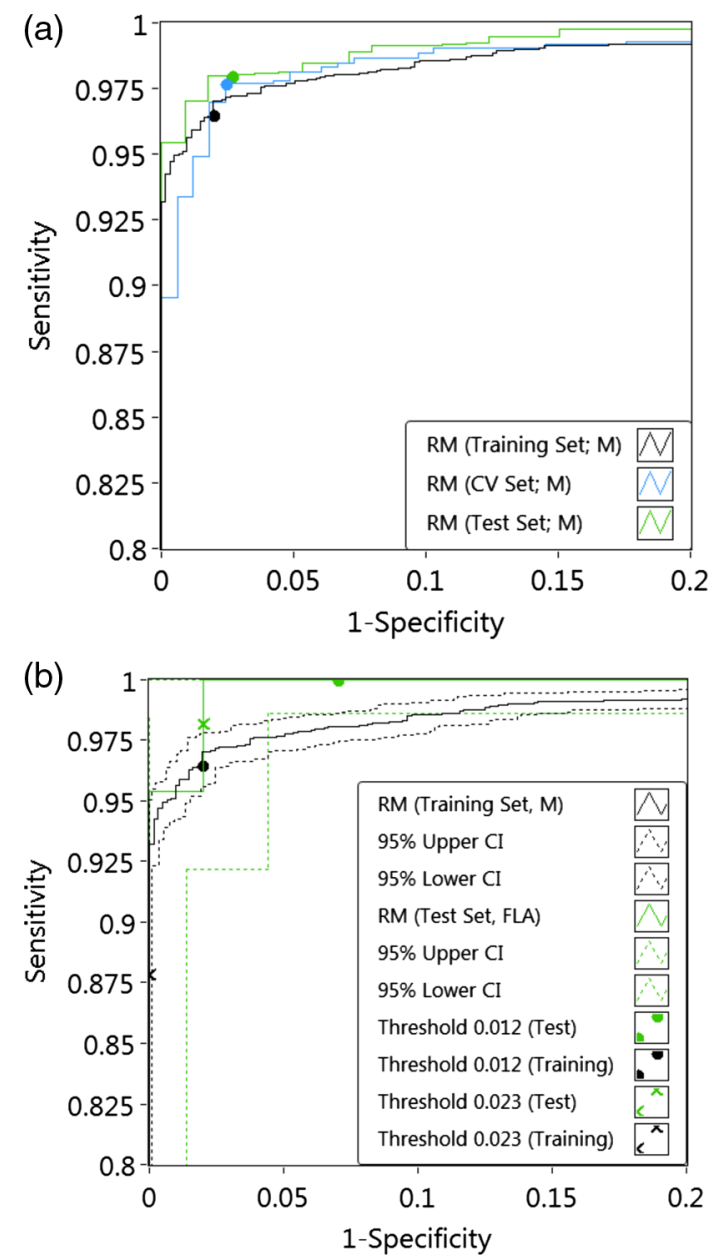

Fig. 11 (a) ROC curve of training, CV, and test datasets with respect to the modulation class (abbreviation "M") only. (b) ROC curve of training and test dataset. The test dataset is plotted with respect to its FLA-visibility class (abbreviation "FLA"). The dotted lines represent the $95 \%$ confidence intervals.

of 0.012 is used. False positives are those spots without any sign of MBF (FLA-nonvisible) but an incorrect algorithmic assignment to the opposite class (FLA visible). A false-positive case occurs if the RM value is above the threshold for an acquired signal, classified as "FLA-nonvisible" for at least one single pulse. These cases are not expected to induce a therapeutic effect. There are seven false-positive pulses originating from five spots and four patients. In all false positive sensor data pulses, modulations can be identified in the sensor data.

Two commonalities of the misinterpreted data have been identified. In the first case, a slight pathologic hyperfluorescence in an FLA image taken before treatment may lead to the wrong conclusion that a hyperfluorescence in the post-treatment image does not originate from laser irradiation at all. In the second case, it is noticeable that modulations in the sensor data do not continue until the end of the pulse. This is unusual for larger microbubbles but more common for laser pulse instabilities of the laser cavity itself. This assumption cannot be tested in the context of this work.

\subsection{Necessity of Microbubble Monitoring}

In this study, the ED50 radiant exposure of FLA visible pulses with highest $\mathrm{RM}$ value is $625 \mathrm{~mJ} / \mathrm{cm}^{2}$. Thus, the ED50 is 
comparable to those found in a previous study ${ }^{30}$ (ED50 $=$ $640 \mathrm{~mJ} / \mathrm{cm}^{2}$ ) for the application of 30 pulses of equal energy.

To deal with interindividual variations of the irradiance for initial MBF, probing methods for adequate treatment energy may be applied. In the results chapter, it is apparent that the pulse-energy-threshold for MBF varies intraindividually up to a factor of 4.4 (see Patient DME2 in Table 4). This complicates the treatment process with regard to probing methods. Probing methods can make SRT applicable, but depending on the exact procedure, the risk of over- and undertreatment can be increased. In the following section, a case (one treatment of one patient) of undertreatment is highlighted. Overtreatments (ophthalmoscopically visible spots) did not occur in this study.

In this treatment, a pulse energy of $E_{\mathrm{Max}}=110 \mu \mathrm{J}$ leads to FLA visibility in the test spot region, but not in the treatment region. Figure 12(a) displays the test spot region of this patient. The green ellipse surrounds the spots irradiated with $E_{\text {Max }}=110 \mu \mathrm{J}$. Figure 12(b) displays the raw data originating from one of these spots. Microbubble-related modulations can be found in this data. Panels (c) and (d) display FLA images of the corresponding treatment region (the same eye) taken before and after treatment, respectively. The treatment spots have been placed close to the region that is already hyperfluorescent in the pretreatment image. The absence of additional hyper fluorescence in the post-treatment image [panel (d)] is congruent with the absence of microbubble-induced modulations in the corresponding raw data [Fig. 12(e)]. The optical microbubble detection technique would have prevented this undertreatment by offering information about the absence of MBF. In the actual treatment protocol, post-treatment FLA images were used to detect such cases.

Figures 12(b) and 12(e) also demonstrate that pulses with microbubble-induced modulations can be lower in amplitude than pulses without microbubble-induced modulations. This can lead to problems in algorithms without normalization procedures. Normalization methods to the backscattered pulses integral, sum, etc. are preferred since these approaches take the most influencing factors (pulse energy, transparency of anterior eye media, etc.) into account. Neglecting an adequate normalization procedure can lead to cases where high amplitude pulses without microbubble-induced modulations can result in $\mathrm{RM}$ values higher than those of small amplitude signals with microbubble-induced modulations. The normalization procedure in the presented algorithm removes this unwanted influence.

\subsection{Interpretation of the Microbubble-Detection- Based Therapeutic Window}

It is apparent in Fig. 7 that RM values of FLA-visible regions can be higher than those of ophthalmoscopically visible regions. Thus, knowledge regarding potential ophthalmoscopic visibility cannot be gained based merely on RM values. As in previous SRT studies, a TW is defined to express the range from FLAvisible to ophthalmoscopically visible effects. Despite the more conservative definition of the microbubble-detection-based $\mathrm{TW}_{\delta}$, it is wider than the classic TW evaluated in former studies for a series of 30 pulses of constant pulses energy (pulse duration $1.7 \mu \mathrm{s}$, repetition rate $100 \mathrm{~Hz}$, spot size $200 \mu \mathrm{m}) .{ }^{30}$ These former studies have defined an interindividual TW in terms of radiant exposure ratios. This causes the lower TW boundaries to be influenced by eyes with a high FLA-visibility threshold energy. These energy levels may be caused by low transparency of the anterior eye media and low RPE pigmentation. Likewise, the upper TW boundaries were influenced by eyes with a low threshold energy for ophthalmoscopic visibility. Lowered upper TW boundaries may be caused by high transparency of the (a)

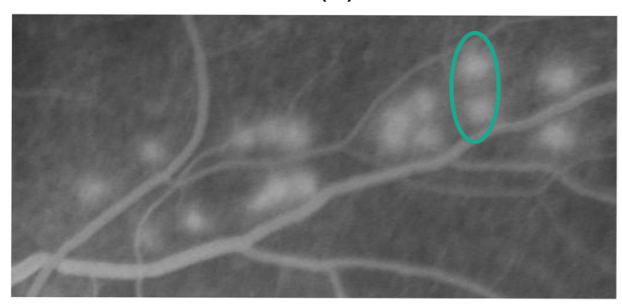

(c)

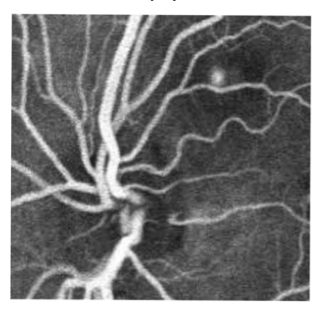

(d)

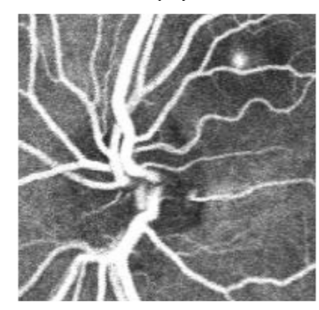

(b)

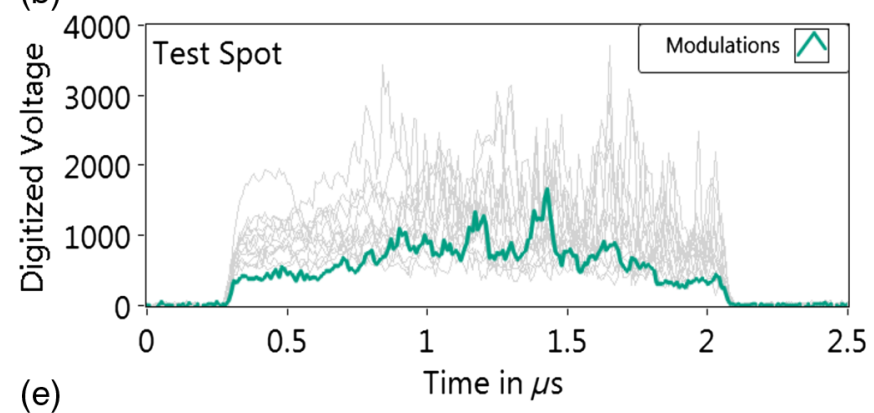

(e)

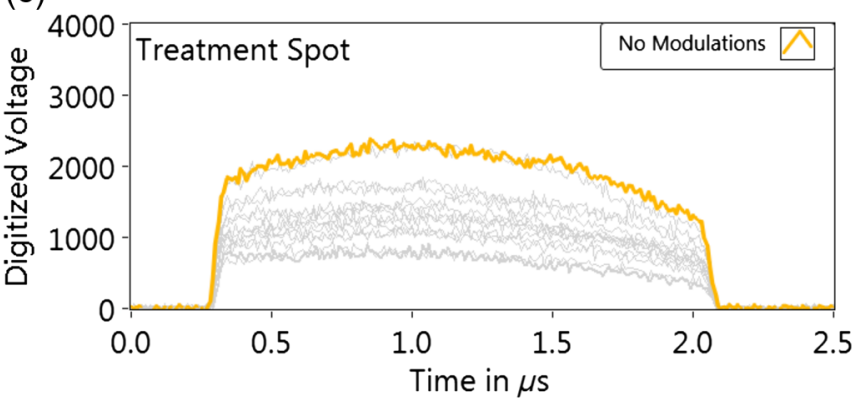

Fig. 12 (a) FLA image taken after treatment. Green ellipse highlights test spots applied with $110 \mu \mathrm{J}$. (b) Sensor data of those spots are displayed. The pulse colored in green is the first pulse of this spot. Microbubble-induced modulations can be seen. (c) and (d) FLA images of the treatment region before and after treatment were shown. (e) An example of treatment pulses applied with $110 \mu \mathrm{J}$ is displayed. Even pulses near $E_{\max }$ do not display microbubble-induced modulations (yellow). 
anterior eye media and high RPE pigmentation. With the presented microbubble detection based definition, this tradeoff is reduced for the microbubble detection-based $\mathrm{TW}_{\delta}$.

The variations in the max-min values of local $E_{\mathrm{MBF}}$ values (see Table 4) emphasize the necessity for a single-pulse microbubble detection technique. Only by such techniques, knowledge about the position inside/outside the $\mathrm{TW}_{\delta}$ can be gained.

Additionally, it should be mentioned that the differences in the ensemble of treated diseases can influence the TW as well. While a former study included CSCR, DME, geographic atrophy, and drusen macluopathy ${ }^{30}$ the present study only includes CSCR and DME.

\subsection{Limitations of the Optical Microbubble Detection Method}

Despite the advantages of the optical microbubble detection technique compared with arbitrary pulse energy selection, several influential factors need to be considered.

Changes in the optical conditions of the anterior eye media may reduce the amount of backscattered light to an extent that makes the evaluation of the signal impossible.

The optimization process was performed for only 15 eyes ( 10 were evaluable by FLA imaging). This leads to uncertainties in the statistical analysis (ROC and Probit). More data, especially more FLA-nonvisible and ophthalmoscopically visible data, are required to reduce these uncertainties. Furthermore, all data belong to a certain subgroup (CSCR and DME patients). For coverage of a larger variety of factors influencing the system's performance, a larger sample size is needed. A spotwise evaluation of the TW was not possible since there were not enough ophthalmoscopically visible spots with the first pulse below the threshold for bubble formation. A lower starting pulse energy $(<50 \%$ of maximum pulse energy) for the ramp can solve this problem in future studies.

\section{Conclusion}

The algorithm introduced here can be applied to all SRT systems using energy ramping. It can not only be used to detect MBF, it can also be used as a real-time automatic feedback control system to cease laser radiation immediately or few pulses after the MBF is passed in order to enable safe and effective irradiation within the lower therapeutic window. At a threshold value of 0.12 , sensitivity and specificity to discriminate microbubbleinduced modulations are 0.98 and a 0.97 , respectively. If the threshold value is increased to 0.23 , no false positives, and thus no undertreatment occurs, which results in a specificity of 1 and sensitivity of 0.88 while still being within the TW.

The technique presented in this work may may be combined with an optoacoustic microbubble detection method to detect MBF since the acoustical approach supports the scenario of blurry anterior eye media. Microbubble detection methods are expected to increase safety, as knowledge is gained about the local MBF threshold, and treatment efficiency through a reduction of the number of retreatments induced by undertreatments. The approach to define a Microbubble-Detection-Based Therapeutic Window $\left(\mathrm{TW}_{\delta}\right)$ can be applied in other microbubble detection techniques based on optoacoustics or OCT as well.

\section{Disclosures}

Ralf Brinkmann is an inventor of patents, who are owned by the Medizinisches Laserzentrum Lübeck $\mathrm{GmbH}$ and licensed to commercial entities, that are related to the technology and analysis methods described in this study. The patients data were acquired and processed by coauthors unaffiliated with any commercial entity.

\section{Acknowledgments}

We thank Lutronic, Inc. (Republic of Korea) for resourcing the R:GEN Laser Systems including the hardware for detection of backscattered light and the according raw data. The Lutronic algorithm for data processing is very different from the algorithm presented here and has neither used nor analyzed at any time.

\section{References}

1. Early Treatment Diabetic Retinopathy Study Group, "Early photocoagulation for diabetic retinopathy. ETDRS report no. 9," Ophthalmology 98(5), 766-785 (1991).

2. Early Treatment Diabetic Retinopathy Study Group, "Photocoagulation for diabetic macular edema. ETDRS report No. 1," Arch. Ophthalmol. 103(12), 1796-1806 (1985)

3. Branch Vein Occlusion Study Group, "Argon laser scatterphotocoagulation for prevention of neovascularization and virteous hemmorrhage inbranch vein occlusion. A randomized clinical trial," Arch. Ophthalmol. 104(1), 34-41 (1986).

4. M. A. Mainster, "Decreasing retinal photocoagulation damage: principles and techniques," Semin. Ophthalmol. 14(4), 200-209 (1999).

5. S. Al-Hussainy, P. M. Dodson, and J. M. Gibson, "Pain response and follow-up of patients undergoing panretinal laser photocoagulation (PRP) with reduced exposure times," Eye 23(2), 491 (2008).

6. M. Figueroa et al., "Laser photocoagulation for macular soft drusen," Retina 17, 378-384 (1997).

7. G. Bresnick, "Diabetic maculopathy: a critical review highlighting diffuse macular edema," Ophthalmology 90, 1301-1317 (1983).

8. J. Roider et al., "Micro-photocoagulation: selective effects of repetitive short laser pulses," Proc. Natl. Acad. Sci. U. S. A. 90, 8643-8647 (1993).

9. R. Brinkmann et al., "Origin of retinal pigment epithelium cell damage by pulsed laser irradiance in the nanosecond to microsecond time regimen," Lasers Surg. Med. 27, 451-464 (2000).

10. C. Framme et al., "Influence of pulse duration and pulse number in selective RPE laser treatment," Lasers Surg. Med. 34, 206-215 (2004).

11. J. Roider et al., "Selective retina therapy (SRT) for clinically significant diabetic macular edema," Graefe's Arch. Clin. Exp. Ophthalmol. 248, 1263-1272 (2010).

12. A. Yasui et al., "Retinal sensitivity after selective retina therapy (SRT) on patients with central serous chorioretinopathy," Graefe's Arch. Clin. Exp. Ophthalmol. 255, 243-254 (2017).

13. Y. J. Kim et al., "Selective retina therapy with real-time feedbackcontrolled dosimetry for treating acute idiopathic central serous chorioretinopathy in Korean patients," J. Ophthalmol. 2018, 1-9 (2018).

14. G. Schuele et al., "RPE damage thresholds and mechanisms for laser exposure in the microsecond-to-millisecond time regimen," Invest. Ophthalmol. Visual Sci. 46, 714-719 (2005).

15. J. Roider et al., "Response of the retinal pigment epithelium to selective photocoagulation," Arch Ophthalmol. 110, 1786-1792 (1992).

16. C. Framme et al., "Structural changes of the retina after conventional laser photocoagulation and selective retina treatment (SRT) in spectral domain OCT," Curr. Eye Res. 34, 568-579 (2009).

17. R. G. Borland, "The role of fluorescein angiography in the detection of laser-induced damage to the retina: a threshold study for Q-switched, neodymium and ruby lasers," Exp. Eye Res. 27(4), 471-493 (1978).

18. R. Birngruber, C. Puliafito, and A. Gawande, "Femtosecond laser-tissue interactions: retina1 injury studies," IEEE J. Quantum Electron. 23(10), 1836-1844 (1987). 
19. M. P. Lopez-Saez et al., "Fluorescein-induced allergic reaction," Ann. Allergy Asthma Immunol. 81(5), 428-430 (1998).

20. D. F. Marcus, J. A. Bovino, and D. Williams, "Adverse reactions during intravenous fluorescein angiography," Ann. Ophthalmol. 15(11), 1084-1086 (1983).

21. E. Seifert et al., "Automatic irradiation control by an optical feedback technique for selective retina treatment (SRT) in a rabbit model," Proc. of OSA-SPIE 8803, 880303 (2013).

22. C. Alt et al., "Selective targeting of the retinal pigment epithelium using an acousto-optic laser scanner," J. Biomed. Opt. 10(6), 064014 (2005).

23. S. Zbinden et al., "Automatic assessment of time-resolved OCT images for selective retina therapy," Int. J. Comput. Assist. Radiol. Surg. 11(6), 863-871 (2016).

24. P. Steiner et al., "Time-resolved ultra-high resolution optical coherence tomography for real-time monitoring of selective retina therapy," Invest. Ophthalmol. Vis. Sci. 56(11), 6654-6662 (2015).

25. G. Schuele et al., "Optoacoustic real-time dosimetry for selective retina treatment," J. Biomed. Opt. 10(6), 064022 (2005).

26. E. Seifert et al., "Toward automated selective retina treatment (SRT): an optical microbubble detection technique," Proc. of SPIE 10474, 104740P (2018)

27. Y. G. Park et al., "Safety and efficacy of selective retina therapy (SRT) for the treatment of diabetic macular edema in Korean patients," Graefe's Arch. Clin. Exp. Ophthalmol. 254(9), 1703-1713 (2016).

28. A. Bradley, "The use of area under the ROC curve in the evaluation of machine learning algorithms," Pattern Recognit. 30(7), 1145-1159 (1997).

29. W. J. Youden, "Index for rating diagnostic tests," Cancer 3(1), 32-35 (1950).

30. C. Framme et al., "Comparison of threshold irradiances and online dosimetry for selective retina treatment (SRT) in patients treated with 200 nanoseconds and 1.7 microseconds laser pulses," Lasers Surg. Med. 40(9), 616-624 (2008).

Eric Seifert received a diploma in medical engineering at the University of Cooperative Education Bautzen and a master of science in photonics at Aalen University. He worked for 7 years at the Medical Laser Center Lübeck $\mathrm{GmbH}$ and (co)authored several papers and conference proceedings on selective retina therapy and other topics on retinal laser treatment.

Jan Tode is an ophthalmologist at the Department of Ophthalmology at University of Kiel. He studied medicine at the University of Kiel and Bialystock (Poland). He completed his ophthalmological training in 2016. He is the head of Murine Experimental Laboratory at the department. His research focuses on translational examinations for the evaluation of new therapeutic aspects in the treatment of uveitis and degenerative retinal diseases.

Amelie Pielen is an ophthalmologist at the University Eye Hospital of Hannover Medical School (MHH). She studied medicine at the University of Bonn, Perugia (Italy), Adelaide (Australia) and Freiburg.
She completed her training in ophthalmology in 2010 at Freiburg and joined Prof. Framme's Team at MHH in 2013. Her research focuses on medical and surgical retina, imaging, and SRT.

Dirk Theisen-Kunde studied engineering at Polytechnic University Lübeck (Germany) and physics at the University of Loughborough (United Kingdom). He worked 6 years at the Institute of Biomedical Optics at the University of Lübeck in the field of endoscopic laser surgery. Since 2010, he is CTO at the Medical Laser Center Lübeck $\mathrm{GmbH}$ and responsible for clinical prototyping of medical devices. His R\&D focuses mainly on ophthalmology and laser surgery.

Carsten Framme is a professor and chairman of ophthalmology at the Medical School of Hannover (MHH) in Germany since 2012. He studied medicine at the $\mathrm{MHH}$ and was clinically trained in Lübeck, Regensburg, and Bern (Switzerland). He did a research fellowship at the Medical Laser Center in Lübeck and at the Wellman Laboratories of Photomedicine in Boston. His research focuses on retinal laser medicine as well as on drug-tissue interactions and surgical approaches.

Johann Roider is a professor of ophthalmology. He studied mathematics, physics, and medicine at Munich Universities, Germany. $\mathrm{He}$ did a research fellowship at the Wellman Laboratories of Photomedicine, Boston. His clinical training was obtained at the Universities of Lübeck and Regensburg. Since 2002, he has been the chairman of the Department of Ophthalmology, University of Kiel. His research focuses are on laser-tissue interactions and vitreoretinal disorders.

Yoko Miura studied medicine in Osaka, Japan, and received a medical license in 1997 and $\mathrm{PhD}$ in 2002. After working in the Osaka City University Hospital till 2006, she engaged in basic research at the University Eye Clinic in Kiel till 2009. She received a German medical license in 2012, and today, she is a faculty member at the Institute of Biomedical Optics and an Ophthalmologist and at the Department of Ophthalmology at University of Luebeck.

Reginald Birngruber studied electrical engineering and physics and graduated in physics (PhD) and in medical biophysics (MD habilis) in Germany. After his retirement from CEO and CRO of the Medical Laser Center Lübeck and the chair of the Institute of Biomedical Optics, he now makes his expertise and contacts available to colleagues in Lübeck and Boston. Current activities include mechanisms of laser tissue effects, optical tissue diagnostics, laser therapy control, and new therapeutic techniques.

Ralf Brinkmann studied physics at the University of Hannover, Germany, with a focus on quantum optics and lasers. After a 5-year industrial interim period, he joined the Medical Laser Center in Lübeck, Germany, in 1993 and received his PhD from the University of Lübeck. Since 2005, he has been holding a permanent position as a faculty member at the University Institute of Biomedical Optics and is also leading the Medical Laser Center as CEO. 